\title{
LA INTRODUCCION DE LA CIENCIA MODERNA EN ANDALUCIA A LO LARGO DEL SIGLO XIX: EL CASO DE SEVILLA
}

José Manuel CANO PAVON*

\section{INTRODUCCION}

Existen pocas noticias en la bibliografía acerca de la introducción de la ciencia moderna en Andalucía. Los estudios realizados se refieren de forma global a la totalidad de España, como los publicados por López Piñero, en los que considera a todas las ciencias de forma conjunta, con un cierto énfasis hacia la Física; la introducción de la Química en nuestro país ha sido abordada de forma específica por Gago Bohórquez. También en el «Diccionario histórico» elaborado por López Piñero y colaboradores se dan datos interesantes sobre este tema, aunque no son muy abundantes las referencias a Andalucía durante el siglo XIX.

Faltan, por tanto, estudios locales referentes a esta cuestión. Sobre el siglo XVIII en cambio existen algunas publicaciones referidas a los centros extrauniversitarios sevillanos, en concreto al Colegio Seminario de San Telmo, a la Regia Sociedad de Medicina y a la Sociedad Patriótica, centros en los que se cultivaban, con fines docentes o de divulgación, algunas disciplinas científicas (principalmente Matemáticas o Física aplicada). En dos publicaciones anteriores del autor de este artículo se han expuesto algunos datos referidos a la Universidad de Sevilla, aunque haciendo una investigación más compleja sobre el siglo XX. En el presente trabajo se ha efectuado un estudio detallado sobre la situación de algunas materias científicas a lo largo del siglo XIX en la capital sevillana, así como sobre la forma en que estos conocimientos científicos se difundieron y evolucionaron. En concreto, se ha considerado a la Física, la Química y las Ciencias Naturales; aunque son tres materias con nna problemática muy diferente, su estudio a lo largo de este período puede servir como

* Catedrático de Química Analítica. Universidad de Málaga. 
indicador para comprender la situación de la docencia y de la muy incipiente investigación durante el siglo XIX en Sevilla y, por extensión y con las debidas matizaciones, en Andalucía.

\section{LA INTRODUCCION DE LA FISICA EXPERIMENTAL}

\subsection{Aspectos generales}

El cultivo de la Física, al igual que el de la Química, fue en España casi exclusivamente docente a lo largo del siglo XIX. Se realizaban experiencias y demostraciones para los alumnos que cursaban estas asignaturas, pero sin efectuarse trabajos que puedan ser considerados propiamente como investigación. Esta penuria contrasta con la actividad desplegada por los naturalistas en este mismo período; quizás la causa de esta mala situación puede haber sido la escasez de medios y de instalaciones para llevar a cabo una mínima labor, problema que no existía para los naturalistas en este mismo período; quizás la causa de esta mala situación puede haber sido la escasez de medios y de instalaciones para llevar a cabo una mínima labor, problema que no existía para los naturalistas, que desarrollaban fundamentalmente una actividad de campo, basada en la observación y en la clasificación más que en la experimentación.

La Física experimental, moderna, tuvo en Sevilla, al igual que en otras zonas de España, grandes dificultades para su introducción. En la Universidad Hispalense existía desde tiempo inmemorial una llamada cátedra de Física escolástica, en la que se explicaba básicamente las obras de Aristóteles; esta situación se mantuvo a lo largo de todo el siglo XVIII con muy escasas modificaciones; al final de la centuria comenzaron a recomendarse algunos textos mas actualizados ${ }^{1}$, a pesar de que en el plan de estudios de Olavide de 1769 se había ya previsto la enseñanza de la Física experimental. Existía indudablemente una oposición sorda de los sectores universitarios más conservadores a la introducción de los conceptos de la Física moderna, que rompían en cierto modo la concepción cosmológica tradicional del mundo, basada en el geocentrismo; por otra parte, no se le daba a los conocimientos físicos la importancia práctica que indudablemente tenían; incluso en los sectores ilustrados las ciencias seguían teniendo un carácter de divertimento, de simple curiosidad intelectual. Esta falta de visión de las posibilidades de las ciencias desaparecería en el siglo XIX con la irrupción de los grandes inventos (comenzando con los buques de vapor y continuando con los ferrocarriles y el telégrafo) que егаn en realidad aplicaciones técnicas, concretas, de los principios de la Física.

1. Entre ellos pueden citarse los libros de Jacquier y de Piquer. 
El atraso de la Universidad, atraso no privativo de Sevilla desde luego, hacía que aquéllas personas interesadas en adquirir algunos conocimientos científicos tuvieran que acudir a los centros extrauniversitarios. En la Sociedad Patriótica, por ejemplo, existía una cátedra de Matemáticas que ocupaba el francés Pierre Henry; también existían cátedras de esta materia en el Colegio Seminario de San Telmo, aunque orientadas básicamente hacia la navegación. En la Regia Sociedad de Medicina se hacían con frecuencia demostraciones de Física, e incluso se había intentado el empleo de la electricidad en la curación de ciertas enfermedades.

La enseñanza de la Física en la Universidad se llevó a cabo por las presiones gubernativas. En el plan de estudios de 1807, conocido como plan de Caballero, se disponía la obligatoriedad de la enseñanza de la Física en la Facultad de Filosofía, como etapa previa para cursar estudios en otras facultades; también era obligatoria la Química para los que fueran a cursar la carrera de Medicina. La enseñanza de dichas asignaturas debía ser diaria, especificándose en el plan los horarios, libros a seguir y organización de las clases. También se preveían clases de Matemáticas, así como de Botánica (esta en la Facultad de Medicina).

La promulgación de este plan, en plena época veraniega, originó en la Universidad sevillana un cambio de asignaturas entre los profesores, encargándose Manuel María del Mármol, catedrático de Filosofía, de las enseñanzas de la Física experimental. Estas mejoras fueron, sin embargo, fugaces, ya que el desencadenamiento de la guerra contra los franceses en la primavera siguiente alteraría de forma importante la vida universitaria; el número de alumnos matriculados descendió espectacularmente, pasándose de 123 matriculados en Física en 1807 a sólo cinco dos años después ${ }^{2}$. El mismo edificio de la Universidad (la antigua casa profesa de los jesuitas) sirvió en ocasiones para alojamiento de tropas ${ }^{3}$ y como taller de pirotecnia para la fabricación de municiones ${ }^{4}$.

El fin de la guerra dejó en muy mala situación a la Universidad y a los centros culturales extrauniversitarios citados anteriormente, a causa de la penuria económica y las depuraciones de los miembros afrancesados. Aunque el plan de estudios de 1807 seguía oficialmente vigente, las universidades seguían rigiéndose por sus antiguos planes. En 1815 se creó en Sevilla una escuela de Farmacia, llamada de San Antonio, pero este centro tuvo una vida muy fugaz y desapareció en poco tiempo.

Durante el trienio liberal se consiguió implantar de nuevo la enseñanza de las ciencias experimentales. Se encargó nuevamente Manuel María del Mármol de las clases de Física, y se nombró catedrático de Química a Francisco

2. Archivo Histórico de la Universidad de Sevilla (AHUS), libro 501.

3. AHUS, libro $969,315$.

4. AHUS, libro 959, 343. 
García Otero, profesor cesante de la desaparecida Escuela de Farmacia. Esta reforma se llevó a cabo en 1821, y duró apenas dos años. La restauración absolutista de 1823 volvió a echar por tierra estas enseñanzas. Dos años más tardes, el nuevo plan de estudios (llamado de Calomarde), manifiestamente reaccionario, en el que según Peset «late todavía el respeto por las viejas universidades y sus ritos, sus pompas vacías de la decadencia», reafirmó la necesidad de la enseñanza de la Física y la Química, aunque no se arbitraron recursos para este tipo de docencia.

En la Universidad sevillana la enseñanza en este período siguió un curso irregular, estando en manos de Manuel María del Mármol la de Física. Sin embargo, no siempre se dieron las clases que el plan de estudios establecía. Así, en 1833, el Inspector General de Instrucción Pública se dirigía al rector de la Universidad manifestándole que «habiendo llegado a entender esta Inspección que en esa Universidad se admite a los escolares a estudiar Medicina con solo tres años de Filosofía elemental por no haber cátedra de Física experimental y Química, ha resuelto que inmediatamente se establezca esta cátedra, en inteligencia que de no hacerlo se suprimiría en ella la mencionada Facultad de Medicina» ${ }^{5}$. El efecto fue fulminante, pues en el claustro reunido a toda prisa una semana después se acordó por unanimidad «que se obedeciera la orden de la Inspección... y que se instalara la cátedra de Física experimental y Química, principiándose las clases desde el día de mañana... y se nombraba al Sr. D. Manuel María del Mármol, a quien encargaba el claustro que, con el celo y la eficacia que tiene acreditados practicase todas las diligencias conducentes a fin de que la cátedra estuviese con la perfección que el plan exige.... ${ }^{6}$.

En cumplimiento del acuerdo se reanudaron a partir de estas fechas las clases de estas materias y se impartieron con mayor regularidad que antes, encargándose el propio Mármol, auxiliado por Diego Fernández Muñoz. Dado que Mármol era catedrático de Filosofía y estaba sólo encargado provisionalmente de la cátedra de Física, la Universidad la sacó a oposición en 1834; para opositar se requería «además del grado de bachiller en Filosofía, haber estudiado otro año de Matemáticas y dos de Ciencias Naturales» ${ }^{7}$. El problema se planteó al formar el tribunal, pues aunque este se estableció de acuerdo con lo dispuesto en el plan de estudios vigente, nombrándose a Manuel María del Mármol, Juan Bautista Maestre y Francisco de Paula Alvarez, lo cierto es que, salvo Mármol, los otros carecían de conocimientos de Física. Las protestas llegaron al Ministerio, por lo que este se dirigió al rector preguntándole «si los censores que se han nombrado para la cátedra de Física experimental y

\footnotetext{
5. AHUS, libro 972, 1.

6. AHUS, libro 948, 154.

7. AHUS, libro 948, 160.
} 
Química carecen de los conocimientos de estas asignaturas, y qué razones ha habido para su nombramiento» ${ }^{8}$. La respuesta de la Universidad no debió ser satisfactoria, pues no tardó el Ministerio en ordenar que «se suspendieran si no están principiados los ejercicios de la oposición a la cátedra de Física experimental y Química, continuando servida por el sustituto» ${ }^{9}$, situación que se prolongaría durante varios años.

La situación continuó siendo precaria, al no existir catedrático en propiedad y al ser muy escasas las asignaciones para la adquisición de instrumentos. En el verano de 1838, por ejemplo, el claustro se lamentaba sobre el estado en que se hallaban las clases de Física y algunas de Medicina, «faltas de máquinas y útiles para dar la enseñanza de una manera digna», que no se correspondían «con los adelantos hechos en estos ramos del saber» 10 .

No sería hasta los primeros años del reinado de Isabel II, y bajo el gobierno de los moderados, cuando se comenzó a realizar una reforma en serio. En 1845 se aprobó un nuevo plan, el llamado de Pidal, en el que se creó el cuerpo de catedráticos de Universidad, reuniendo en un mismo escalafón a los poco más de doscientos catedráticos existentes (por lo general, de origen muy variado, ya que junto a los de las universidades se agregaron otros procedentes de diversas escuelas de Cirugía, Farmacia, etc.); se dotaron nuevas cátedras para las asignaturas experimentales y se creó en las facultades de Ciencias, aunque la mayoría de ellas, como la de Sevilla, solo impartirían los cursos preparatorios de acceso a las otras facultades y escuelas de ingenieros.

En la Universidad de Sevilla, entre 1845 y 1847 se crearon varias cátedras científicas: Mineralogía y Zoología, y Matemáticas, y se desdobló la de Física en dos: Física General y Química. La de Física la ocupó en 1846 el médico sevillano Fernando Santos de Castro, que había estado encargado de las enseñanzas de forma provisional desde varios años antes. Este profesor la desempeñó hasta su fallecimiento en 1890.

En 1850 comenzó a funcionar la Escuela Industrial Sevillana. Este centro, que pudo haber tenido una gran importancia en el desarrollo industrial andaluz, se instaló en un edificio de la calle Amor de Dios, y llegó a contar con unas buenas instalaciones para su época ${ }^{11}$, así como con una excelente, aunque incompleta, plantilla de profesores, entre los cuales puede citarse al catedrático de Física, el ingeniero catalán Ramón Manjarrés Bofarull. Este profesor pasaría años más tarde a la Facultad de Ciencias. La Escuela Industrial cerró

\footnotetext{
8. AHUS, libro 972, 34 .

9. AHUS, libro 972,50 .

10. AHUS, libro $948,212$.

11. La información sobre la Escuela Industrial sevillana se hallan en AHUS, principalmente en los legajos 568 a 579, aunque la documentación que estos contienen se halla completamente desordenada, posiblemente por el poco cuidado que se puso en recogerla al cerrarse el centro.
} 
sus puertas en 1866, perdiéndose así durante un siglo la posibilidad de contar con una escuela de ingenieros en Andalucía.

\subsection{Manuel María del Mármol y su obra científica}

Expuesto ya de forma esquemática los aspectos generales de la azaroza evolución del cultivo de la Física en la Sevilla decimonónica, se van a considerar las aportaciones individuales de algunas de las personas que contribuyeron a su difusión.

Manuel María del Mármol fue el pionero de la docencia de la Física en la Universidad de Sevilla, partiendo de cero y en un ambiente no excesivamente favorable. Nacido en Sevilla en 1769 , perteneció por edad y afinidad a la inquieta y brillante generación de Lista, Reinoso, Arjona y Blanco White, generación que influyó apreciablemente en el desarrollo de las letras sevillanas en los últimos años del siglo XVIII y primeros del XIX. Era una personalidad polifacética, casi renacentista. Sacerdote, llegó a ser capellán real, puesto que alternó con la cátedra de Filosofía de la Universidad Hispalense desde 1799. Además de una mediocre obra poética, escribió varias obras científicas que más adelante comentaremos, así como otras de Lógica, de Derecho penal e incluso de taquigrafía (fue de hecho el que introdujo este sistema de escritura rápida en Andalucía). Características destacadas de su obra son su amplia formación humanística y su fuerte motivación pedagógica, aunque no llegara a profundizar en los aspectos concretos de la ciencia.

Mármol debió adquirir necesariamente sus conocimientos científicos en Sevilla, pues prácticamente en esta ciudad residió durante toda su vida, salvo el período de cinco años (1794-99) en que ocupó la plaza de capellán real en Granada. Su formación debió realizarse a través de las lecturas de los libros de Física, como el de Jacquier y el más completo de Piquer. Mármol disponía con bastante probabilidad de una buena biblioteca; a este respecto refiere Blanco White que:

«(Mármol) era un joven sobrio y trabajador, cuatro ó cinco años mayor que yo, muy amable y comunicativo. Su deseo de saber era grande, y así ha seguido toda su vida. Puede decirse con toda objetividad que su única ambición era mejorar el plan de estudios de la Universidad de Sevilla. Estaba siempre dispuesto a comunicar lo que sabía... Mármol me enseñó Geografía y ...puso en mis manos el Organón de Bacon, libro que solo él conocía en toda Sevilla...» ${ }^{12}$.

12. GARNICA (1988), 44 . 
Mármol era un antiaristotélico convencido. En sus escritos, aún reconociendo la importancia del filósofo griego, arremete contra los que en su nombre ejercieron una auténtica dictadura intelectual e impidieron el desarrollo de nuevas ideas, alabando en cambio «a los hombres perspicaces que abatieron la tiranía de los aristotélicos». De él decía Alberto Lista que:

«...fue el primero que ascendiendo a las cátedras de Filosofía declaró la guerra a muerte al antiguo método de descubrir la verdad; el primero que sustituyó en la enseñanza de la Lógica y de la Metafísica las ideas a las voces, el análisis profundo a la nomenclatura estéril de los peripatéticos, el buen gusto y la razón a la barbarie y a los gritos; el primero que dió a conocer en las cátedras de aquella ciencia los principios luminosos de Condillac...» ${ }^{13}$.

Una lectura detallada de sus escritos permite ubicarle dentro del cartesianismo. Tiene una evidente preocupación por el método, aunque le da poca importancia a la Lógica formal. De haber vivido unos años después hubiera sido un admirador de la Lógica matemática, ya que según el testimonio de un contemporáneo, «impuso a sus alumnos de Filosofía como una obligación el estudio de las Matemáticas, que antes sólo se aconsejaba con cierto desdén y a condición de posponerlas siempre a las teorías de las figuras y modos de los silogismos...».

Su primer libro de carácter científico es el dedicado a los barcos de vapor, obrita dirigida a un público más amplio que el de los alumnos universitarios. La realización de este libro, a raíz de la entrada en servicio del «Real Fernando», posiblemente el primer barco de vapor andaluz, que en unas pocas horas hacía el recorrido entre Sevilla y Cádiz, tuvo por objeto exponer de forma sencilla los principios generales de la máquina de vapor diseñada por Watt. Describe inicialmente los fundamentos físicos de la propulsión a vapor:

«Cuando se une el calórico con partes de agua, a cuya mezcla se ha dado el nombre de vapor por los físicos, se enrarecen tanto las partes de agua, que llegan a tener un volumen mil trescientas o mil cuatrocientas veces mayor...» (actualmente sabemos que 1 litro de agua al vaporizarse ocupa 1244,4 litros en condiciones normales, cifra próxima al dato que expone Mármol, de acuerdo con la bibliografía de que disponía)» ${ }^{14}$.

13. COZAR y LISTA (1841), 4 .

14. MARMOL (1817), 8-9. 
A continuación expone los detalles de la máquina, con descripción de los pistones, válvulas, bombas y demás accesorios. La obrita, aparte de su interés científico, es un auténtico canto al progreso, del que Mármol es un decidido partidario:

«...no dependen ya nuestros viajes de la inconstancia del viento, de la variedad de las corrientes, de la complicación de las maniobras. No es ya la ventura quien nos lleva sobre las aguas: es un agente, el más poderoso de la naturaleza, y que depende de nuestras manos disminuir o aumentar...» ${ }^{15}$.

Recuerda asimismo a los que tienen prevención por el nuevo buque los largos y alucinantes viajes a Sanlúcar de Barrameda desde Sevilla, en los que a veces se empleaban hasta más de una semana en los barcos de vela por falta de viento, con interminables paradas en Coria o en mitad de las entonces desiertas marismas, con calor asfixiante o con frío intenso, pasando a veces hambre cuando el viaje se prolongaba más de lo previsto.

Mármol publicó entre 1825 y 1840 una pequeña serie de obras de carácter científico, especialmente textos de Física y pequeños libros de divulgación. Estas obras permiten tener una idea medianamente precisa sobre sus conocimientos científicos, así como sobre las creencias populares que con algunas de dichas obras trataba de corregir. Después de la aparición de su libro sobre los barcos de vapor publicó, en 1825, un folleto combatiendo los rumores que corrían por Sevilla acerca de la aproximación de la Luna a la Tierra. En él confiesa los motivos que le han impulsado a escribirlo:

«...No sé cuando empezó a correr por Sevilla la voz de que la Luna se había acercado a la Tierra; sólo sé que en mi cuarto, en la calle, en la Iglesia, y en cuantas partes estoy, creyendo que puedo hablar algo sobre esta materia, o porque pertenece a la facultad que enseño, o porque he escrito algo de Astronomía, no me dejan vivir, preguntándome toda clase de personas qué juzgo y qué se puede temer de la aproximación a la Tierra que dan supuesto ha sufrido la Luna. Entre los consultantes hay personas más o menos pusilánimes; todas manifestaban miedo... He inferido, por lo que me han dicho, estar demasiado esparcido el rumor que corre, que ha producido muchas alarmas... El evitar estos daños me mueve a tomar la pluma para hacer el bien... único norte que he tenido siempre en mis trabajos todos...» ${ }^{16}$.

15. MARMOL (1817), 37.

16. MARMOL (1825), 3-4. 
A continuación, tras analizar las fuentes del rumor, explica la imposibilidad del mismo basándose en cálculos matemáticos, con los que llega a la conclusión de que si la Luna se hubiera aproximado, el mes lunar se habría reducido sensiblemente, ya que tardaría menos tiempo en recorrer su órbita. Y el hecho de no ser así probaba la falsedad del rumor.

Sus ideas de Física están expuestas de forma más detallada en sus dos libros sobre esta materia: el primero de ellos, titulado «De Re Physica» está escrito en latín y en forma de diálogos, apareciendo publicado en 1827; el segundo, en castellano, se publicó siete años más tarde. Ambos son muy parecidos, salvo en la estructura, aún cuando el segundo presenta mayor interés, al dar mayores detalles e incluir demostraciones matemáticas. Lo escribe «para fijarles a los alumnos el orden de sus explicaciones y hacerlas reducirlas a un texto para que las retengan con mayor facilidad». Consta de 48 capítulos cortos; en los 37 primeros, de mayor carácter teórico, trata de los principios generales de la Mecánica: inercia, leyes generales del movimiento, choques de los cuerpos, etc. En los últimos capítulos trata de diversos dispositivos mecánicos: polea, balana, ruedas dentadas, etc. Si se compara con otras obras de la época, se pueden observar grandes parecidos, lo que prueba que su bibliografía estaba bastante actualizada. En algunos aspectos más delicados evita el pronunciarse abiertamente; así, al hablar de la naturaleza de la materia, y aún cuando ya Dalton había formulado en 1808 su teoría atómica, dice:

«Nada diremos de la eterna cuestión sobre si la materia se concibe infinitivamente divisible, porque es cuestión de nombre e inútil para la Física, y además es propia de los metafísicos, que quieren malgastar el tiempo precioso...» ${ }^{17}$.

En conjunto, su libro no presenta mucha profundiad. Ofrece lagunas considerables en el tratamiento de las ideas de calor y electricidad, así como en estática y dinámica de fluidos. No prodiga en demasía los cálculos algebraicos; muchos de los razonamientos son casi exclusivamente geométricos. Es posible que prefiriera el método geométrico por ser más intuitivo, o bien porque - como es fácil suponer- los conocimientos matemáticos de sus alumnos no fueran muy elevados.

Como obras menores de carácter divulgativo están unos pequeños apuntes de Química y un discurso referente a las pruebas de la validez del sistema astronómico de Copérnico, editados ambos el mismo año de su muerte (1840). También puede citarse un curioso folleto en el que explica en verso dicho sistema astronómico para ayudar a los alumnos a memorizar los datos de distancias y tamaños de los astros.

17. MARMOL (1836), 16. 
Relacionado con la exposición del sistema copernicano está el comentario que escribió en 1827 a una traducción que había realizado él mismo de la obra de Libes sobre el mundo físico. El comentario está escrito en un tono muy cauto, posiblemente por el ambiente represivo existente:

«...Hoy y antes de hoy, todos o casi todos los físicos de Europa sostienen como efectivo el movimiento real de la Tierra. Es seguido el sistema copernicano por numerosos autores católicos, y se enseña dentro de la misma Roma, a vista y ciencia del Papa, del Colegio de Cardenales, de otros muchos ilustres y doctos eclesiásticos... y del tribunal de la fé que hizo abjurar del sistema a Galileo... Pudo ser escandalosa en un tiempo y ofensiva a los oidos piadosos aquella doctrina, y hoy que se sabe no es tan común que escandalize a las personas... En España, una de las naciones más tardas en admitir este sistema, se enseñan autores plenamente copérnicanos... ${ }^{18}$.

Estos párrafos producen asombro en el lector de hoy, y ponen de manifiesto el pobre ambiente intelectual de la Sevilla fernandina, concordando bien con la opinión negativa que expone Blanco White en su autobiografía, aunque esta se refiriera a unos años antes. A doscientos años de la formulación de la teoría heliocéntrica todavía era necesario hablar con cautela de ella, para evitar suspicacias y malos entendidos.

\subsection{Fernando Santos de Castro y su obra docente sobre Física}

Si Mármol corresponde a una época de transición desde la Universidad del Antiguo Régimen a la liberal, y desde el aristotelismo a la ciencia moderna, Santos de Castro (nacido en Sevilla en 1809) es ya un típico profesor decimonónico, con una mejor formación científica. Había cursado la carrera de $\mathrm{Me}$ dicina, por lo que sus conocimientos de Física y Química tuvo que aprenderlos fuera de las aulas universitarias, posiblemente en la Sociedad Patriótica y del propio Mármol, del que fue colaborador durante algún tiempo, y al que sustituiría más tarde. Como catedrático de la Universidad, su preocupación más destacada fue el de construir un gabinete de Física y Química, para lo que tuvo que vencer serias dificultades. Así, en 1849, en un informe a la superioridad, se lamentaba de que:

18. LIBES (1827), 182-184. 
«...Son tan escasos e imperfectos los instrumentos que existen en el gabinete de Física que pueden aplicarse a este objeto (a la observación metereológica) que apenas merecen enumerarse en el estado actual de la Meteorología... El gabinete de esta Universidad sólo cuenta como instrumentos meteorológicos con un mal barómetro con un pequeño termómetro unido, y el higrómetro de Saussure, pues el barómetro de Gay-Lussac y el higrómetro de Daniel, que vinieron entre la colección de instrumentos destinados a esta corporación llegaron rotos desde $\mathrm{Pa}$ rís...» ${ }^{19}$.

aunque años más tarde, y de acuerdo con la reseña de Martín Villa, la dotación del gabinete había aumentado considerablemente.

Básicamente, las obras científicas de Santos de Castro son libros de texto dirigidos a los alumnos. Primeramente tradujo el libro de Ajasson de Grandsaque sobre Física y Meteorología. En el prólogo hace el profesor sevillano unos comentarios muy significativos acerca del estado actual de la enseñanza de la Física en su época, en especial de la escasez de conocimientos matemáticos de los alumnos que llegaban a la Universidad. También se lamenta de que los alumnos no consideren a las ciencias experimentales con la importancia que tienen en sí mismas:

«...De esta forma, los elementos de la Física se ponen al alcance de la juventud, que sólo procura en las clases adquirir nociones generales de las ciencias, bien como una útil o necesaria preparación para estudios superiores, como la Historia Natural ó la Medicina, ó bien solamente como complemento de la educación... ${ }^{20}$.

Seis años más tarde publicó su primer libro original de Física, en dos volúmenes, con una distribución de materias parecida a la de la obra anterior. En la introducción vuelve a lamentarse del estado de las enseñanzas científicas:

«...Desgraciadamente, en nuestro país no ha llegado la ciencia física a ese grado de perfeccionamiento (con respecto a los países europeos), porque ni poseemos medios experimentales abundantes ni hemos hecho las aplicaciones ventajosas de estos preciosos conocimientos a las artes, la industria y las fabricaciones, que dan a los pueblos científicos esa riqueza y preponderancia que constituye la verdadera prosperidad de las naciones...» ${ }^{21}$.

19. AHUS, libro 974, 271.

20. AJASSON DE GRANDSAQUE y FOUCHE (1840), 3-5.

21. SANTOS DE CASTRO (1846), 4. 
Por lo que respecta al contenido del libro, lo mas llamativo para un lector actual es el considerar a la gravitación, la cohesión y la afinidad como manifestación de una misma fuerza, que el denomina la «atracción». Hoy día sabemos que la atracción entre átomos y moléculas no se deben a atracciones gravitatorias (fuerzas muy débiles en relación con las interacciones electromagnéticas), pero en su época era algo frecuente, aunque ya algo anticuado, el intentar agrupar todas las fuerzas físicas en una sola, quizás por la formación cartesiana de algunos autores, que les hacía buscar la clasificación y sistematización de forma preeminente.

\section{LA DIFUSION DE LA QUIMICA}

\subsection{Generalidades}

La Química fue mucho menos cultivada que la Física en la Sevilla del siglo XIX. Esto también había ocurrido durante el siglo XVIII, donde en la Regia Sociedad de Medicina se hacían con mayor frecuencia demostraciones de Electricidad y Mecánica que experimentos químicos. Algo similar ocurría en los otros centros culturales sevillanos. Los esfuerzos que algunos particulares y diversos organismos hicieron para que se creara una cátedra de Química en la Universidad resultaron infructuosos. El establecimiento de la escuela de Farmacia de San Antonio en 1815 pudo ser importante para los conocimientos químicos, pero el centro tuvo una vida demasiado fugaz. Por otra parte, los profesores que ocuparon la cátedra de Química de la Universidad de Sevilla, creada en 1845 , no parece que estuvieran a la altura de sus colegas de Física o de Historia Natural. No publicaron, por ejemplo, ningún tipo de escritos científicos de los que haya quedado constancia. El hecho mismo de que Santos de Castro, catedrático de Física, publicara un libro de texto de Química, parece confirmar la escasas actividades de los titulares de la cátedra de Química. hacia el final de siglo dicha cátedra fue ocupada por Federico Relimpio y Ortega, cuyas inquietudes le hicieron escribir varios libros sobre su disciplina y a gestionar la creación en la Universidad de Sevilla de una sección de Químicas, que comenzó su funcionamiento en 1910.

\subsection{Los primeros profesores de Química: García Otero y Campelo}

Francisco García Otero, el primero de los que ejercieron de catedrático de Química, había sido inicialmente profesor de esta materia en la escuela de

22. El escalafón de catedráticos puede verse en la Gaceta de Madrid de 25 de mayo de 1846. 
Farmacia. Largo tiempo cesante, estuvo adscrito durante el trienio liberal a la enseñanza universitaria, pero cesó de nuevo en 1823 . Cuando en 1845 se aprobó el plan Pidal fue nombrado catedrático de Universidad. Por aquel entonces era ya de edad avanzada. Se jubiló en 1849, siendo decano de la Facultad de Filosofía, sustituyéndole Campelo en la cátedra.

Juan Campelo Ayuevar, nacido en 1814 en Umbrete, corazón de la comarca sevillana del Aljarafe, fue sacerdote, doctorándose en Teología en 1840. Con anterioridad había sido profesor del seminario de Sanlúcar de Barrameda, donde impartió clases de Algebra y Geometría. En 1843 marchó a París, donde permaneció tres años siguiendo cursos de Mineralogía, Química, Botánica, Fisiología y Farmacia. No dejó escritos científicos de interés, lo que sorprende en un hombre que debía tener una buena formación científica tras su larga estancia parisina. Es posible que su actividad de sacerdote limitara su labor científica. Esta labor eclesiástica fue intensa, interviniendo en las sesiones del Concilio Vaticano ${ }^{23}$.

\subsection{Las obras químicas de Fernando Santos de Castro y Ramón Manjarrés}

Santos de Castro publicó en 1845 un libro de texto sobre Química, en dos volúmenes, en el que puede verse el nivel de los conocimientos de esta disciplina en la Sevilla de mediados del siglo XIX. Es curiosa la clasificación que hace de los elementos químicos (a los que llama cuerpos simples); así distingue entre imponderables o fluidos (lumínico, calórico, eléctrico y magnético) y ponderables (metales, metaloides y dudosos). El incluir a la electricidad, la luz, el calor y el magnetismo entre los elementos era ya en su época un claro anacronismo, lo que pone de manifiesto que se hallaba algo anticuado con respecto a la evolución de la Química en ese momento. También tiene algunas definiciones bastante confusas, como por ejemplo la que da del fenómeno de la isomería:

«Cuando dos cuerpos con la misma composición centesimal difieren en sus propiedades químicas serán isoméricos, y en ellos tienen una colocación diferente los átomos compuestos que constituyen su átomo químico».

También cultivó preferentemente los temas químicos el sucesor de Santos de Castro en la cátedra, el catalán Ramón Manjarrés y Bofarull (1828-1918). Con anterioridad había sido profesor de la Escuela Industrial sevillana, e incluso director de la misma. Al desaparecer este centro pasó al de Barcelona,

23. AHUS, libro 1150, 234. 
incorporándose a la Universidad de Sevilla en 1891. Publicó en 1860 un libro de texto sobre Química industrial, abordando preferentemente los procedimientos metalúrgicos, entonces en un claro proceso de expansión en toda Europa. Poco después publicó un folleto sobre el efecto de los fosfatos terreos (principalmente del fosfato cálcico) sobre los vegetales, en el que describe los beneficios que el abonado con estos productos puede producir sobre la producción de cereales, preocupación secular de los españoles. Años más tarde (1896) escribió un libro sobre la producción y refinación del aceite de oliva, en el que combate muchos de los procedimientos seguidos por los olivareros en la obtención del aceite, como el almacenamiento durante un tiempo excesivo de las aceitunas, que provocaba la fermentación de estas, por lo que el aceite que se obtenía de ellas no reunía las debidas condiciones para el consumo.

Otra publicación de Manjarrés, realizada en colaboración con Germán Losada, también profesor de la Escuela Industrial, es una memoria sobre la exposición internacional celebrada en Londres en 1862, en la que estuvieron ambos como comisionados por la Diputación provincial sevillana, «para estudiar dicha exposición en beneficio de la provincia». En la memoria, de más de trescientas páginas, pasan revista a los diferentes produetos expuestos, clasificándolos en diferentes grupos: metalurgia y productos metalúrgicos, productos agrícolas y materias primas, industrias químicas, artes de construcción, industrias mecánicas e industrias lanera y cañamera. Se lamentan en la memoria del poco espacio ocupado por los productos españoles (540 metros cuadrados, frente a los 56.000 de Gran Bretaña y a los 14.000 de Francia). En la exposición se exhibían «muestras de hierros y aceros de los altos hornos del Pedroso, que funcionan con carbón vegetal», altos hornos que desaparecerían poco después al no poder competir con la siderurgia vasca, que empleaba carbones minerales y producía un hierro mucho más barato.

\section{LOS NATURALISTAS SEVILLANOS. LA DIFUSION DEL DARWINISMO}

\subsection{Generalidades}

El cultivo de la Historia Natural en sus diferentes ramas fue sin duda la más brillante actividad científica desarrollada en Sevilla y en Andalucía a lo largo del siglo XIX. Esto es perfectamente explicable por los siguientes motivos: 1) la tradición naturalista española durante el siglo XVIII, con figuras tan importantes como Mutis y Cavanilles, que había dado origen a diversas expediciones a varias regiones de América, 2) la nula oposición que los estudios naturalistas 
habían encontrado en los sectores más conservadores, en contraposición a los trabajos de los físicos y los químicos, 3) el ser una investigación más barata, ya que los naturalistas (geólogos, botánicos, zoólogos) apenas necesitaban medios instrumentales para el desarrollo de su labor, era una investigación «más de botas que de batas», 4) el interés que despertaba Andalucía para los naturalistas foráneos, una región extensa y poco conocida, por su lejanía, las difíciles condiciones orográficas de su zona oriental y la inseguridad de sus caminos, así como también por poseer una flora con evidentes puntos de contacto con la del norte de Africa. Esto explica que fueran numerosos los naturalistas extranjeros que recorrieron las diferentes comarcas andaluzas a lo largo del siglo XIX: geólogos como Du Verneuill, Collomb, Falconer, Delanoue y Lubbock, botánicos como Webb y Boissier, y zoólogos como Sannders, Yrbing y Steindachner.

Otro factor importante que influyó en el desarrollo de los trabajos naturalistas fue la creación de dos cátedras en la Universidad de Sevilla: una de Botánica (que inicialmente estuvo vinculada al Jardín de Aclimatación) y otra de Mineralogía y Zoología, aunque más tarde sólo quedaría esta última, a la que habitualmente se le denominaba de Historia Natural.

En la segunda mitad del siglo XIX los estudios naturalistas vieron incrementarse su interés, a causa del revuelo producido por la difusión de las ideas evolucionistas de Charles Darwin, difusión que fue bastante rápida en Andalucía, debido sobre todo a la labor desarrollada por el entonces catedrático de Historia natural en Sevilla, el gaditano Antonio Machado Núñez, figura importante dentro de los naturalistas andaluces, al que mas adelante se le dedicará una atención especial.

\subsection{Los botánicos: Boutelou y Colmeiro}

La Botánica había sido cultivada en la Sevilla del siglo XVIII gracias al interés que por ella mostró la Regia Sociedad de Medicina, la cual llegó a contar con un modesto jardín botánico y con un especialista encargado del mismo. El primero que desempeñó este puesto fue el farmacéutico Antonio Ramos, al que sustituyó en 1786 el catalán Pedro Abad, que estuvo hasta su fallecimiento en 1800. En el jardín se llegaron a contabilizar más de trescientas plazas diferentes. Abad elaboró asimismo un herbario que contenía unas dos mil especies, y que junto con el de Boutelou pasaría a mediados del siglo XIX a la Universidad.

Tras la muerte de Abad, seguida por la crisis de 1808, la actividad botánica sevillana quedó prácticamente extinguida. Sólo en 1825 , y por iniciativa del asistente Arjona, comenzaron a realizarse algunos trabajos de aclimatación de 
plantas americanas en los llamados jardines de las Delicias. Estos trabajos fueron llevados a cabo por Claudio Boutelou (1774-1842), botánico de origen francés que tras el jardín del Alcázar de Sevilla. La finalidad de estos intentos de aclimatación era el de ensayar el cultivo de plantas de origen americano (principalmente antillano) con fines económicos, así como el de dotar de plantas ornamentales a los jardines sevillanos.

En 1832, tras dichos trabajos preliminares, se acometió en serio la organización de un jardín de aclimatación, dotándolo de una cátedra de Botánica, de la que se encargó Boutelou. El jardín se comenzó a instalar en los jardines del Palacio de San Telmo (actual parque de María Luisa), pero el proyecto no llegaría a concluirse. La cátedra en cambio siguió funcionando regularmente, dándose las clases en la llamada Casa de los Patos de los jardines de las Delicias; tenían un marcado carácter práctico, gracias al vivero anexo. Hacia los años cuarenta comenzó a impartirlas Pablo Boutelou, hijo de Claudio, el cual sustituiría a su padre al frente de la misma a partir de 1842 . La reforma de Pidal de 1845 integró esta cátedra en la Universidad de Sevilla, pasando Pablo Boutelou a propietario de la misma hasta su fallecimiento en 1846.

La cátedra fue ocupada en 1847 por el botánico gallego Miguel Colmeiro Penido (1816-1901), que estuvo al frente de ella hasta su traslado a Madrid en 1857. Colmeiro fue un naturalista muy activo; en los diez años que permaneció en Sevilla realizó un trabajo sobre la madera de alerce y otro sobre las encinas; también hizo una revisión de los escritos del botánico Rojas Clemente sobre los líquenes andaluces. Ya en Madrid publicó varias obras fundamentales de síntesis sobre la flora ibérica y sobre los botánicos españoles.

La influencia de Colmeiro en la Botánica española fue considerable. Fue el primero que aplicó en España el método de clasificación natural, sustituyendo al ya anticuado sistema sexual de Linneo. En Madrid, además de desempeñar su cátedra, fue director del Jardín Botánico. Durante su estancia en Sevilla se preocupó asimismo de incrementar los antiguos herbarios de Abad y Boutelou. Sin embargo, a pesar de toda su actividad, no llegó a crear escuela en la ciudad; a su marcha la cátedra de Botánica desapareció, aunque sus enseñanzas continuarían, impartidas por Antonio Machado Núñez, que ocupaba la cátedra de Mineralogía y Zoología desde 1846. Los trabajos botánicos se interrumpieron de todas formas durante varios años, ya que Machado cultivó preferentemente las materias específicas de la asignatura de la que era titular.

\subsection{Machado Núñez y su labor científica}

La figura de Machado Núñez es especialmente atractiva, tanto por su singular personalidad humana como por la de sus descendientes; fue padre de An- 
tonio Machado Alvarez, antropólogo e iniciador en Andalucía de los estudios sobre el folklore, y tuvo como nietos a los poetas Manuel y Antonio Machado Ruiz, cuyas famas han oscurecido injusta e involuntariamente a la del abuelo.

Machado nació en Cádiz, posiblemente en 1815, siendo su familia oriunda de Huelva. Hizo los estudios de Medicina en su ciudad natal, licenciándose en 1838. Residió casi dos años en Guatemala ejerciendo su profesión médica. A su regreso marchó a Paris, donde siguió los cursos de Química de Dumas y Orfila, y los de Geología con Constant Prevost. A su vuelta a España se estableció en Sevilla, vinculándose a la Universidad. Tras las correspondientes oposiciones obtuvo una cátedra en Santiago de Compostela, y desde allí pasó al poco tiempo a la capital andaluza (1846). En la Universidad sevillana permaneció hasta 1886, en que al filo de los setenta años se trasladó a la de Madrid.

La época de mayor actividad de Machado es la comprendida entre 1865 y 1875. Aparte de su labor docente y de sus artículos de divulgación, ocupó numerosos cargos: decano de la Facultad de Filosofía y de la de Ciencias, rector de la Universidad y gobernador civil. Fundó con el filósofo Federico de Castro la llamada «Revista mensual de Filosofía, Literatura y Ciencias de Sevilla», que apareció regularmente entre 1869 y 1874, y en la cual publicó numerosos artículos científicos; en esta revista confluyeron las tres corrientes ideológicas más progresistas que se daban en aquel momento en la ciudad: el hegelismo, el krausismo y el positivismo. En esta última es donde puede ubicarse a Machado. Su fé en los datos experimentales, su optimismo en la capacidad de progreso del hombre y su sustitución mental de los principios religiosos por los científicos no admite duda sobre su localización ideológica. Así, llega a afirmar en uno de sus artículos que:

«Nosotros negamos la intervención sobrenatural de la manera que algunos místicos la presentan; sería absurdo admitirla aún para aquéllos hechos de que no podamos dar una explicación plausible, pues la ignorancia de las causas productoras de un fenómeno, de ninguna manera debe destruir nuestra razón que nos dice que está todo sujeto a leyes más o menos conocidas; a la ciencia pertenece descubrirlas por medio de la observación y la experiencia, y mientras tanto no deben aceptarse puerilidades inconvenientes... ${ }^{24}$.

Otra de las realizaciones de Machado fue la creación de la Sociedad Antropológica de Sevilla en diciembre de 1871. En la sesión inaugural de la misma pronunció un discurso sobre los aspectos más destacados de esta por entonces nueva ciencia:

24. MACHADO (1872b), 528. 
«La antropología es la ciencia del hombre, es el conocimiento de su ser material, considerado como la síntesis de todos los organismos animados; es la historia de los adelantos que la civilización ha hecho en todas sus variedades y razas; es el estudio de sus facultades morales y psicológicas... en una palabra, la antropología nos da a conocer la historia física, social y filosófica de nuestra especie, unida en estrecho lazo, confundida en las grandes cuestiones fundamentales y caminando de común acuerdo para alcanzar la noción de sí mismo de una manera más completa» ${ }^{25}$.

Los miembros fundadores de dicha sociedad eran médicos en su mayoría (Vicente Chiralt, por ejemplo), así como profesores universitarios (Federico de Castro, Rafael Caro, etc.). En las reuniones que celebraban periódicamente se trataban cuestiones biológicas, sociológicas (como la igualdad del hombre y de la mujer en el matrimonio), filológicas (como los mecanismos del lenguaje) y costumbristas.

Machado prodigó más el artículo divulgativo que la obra extensa. Aparte de algunas traducciones, sus libros originales editados son cuatro catálogos sobre la fauna andaluza (especialmente de la zona occidental) que aparecieron bastante distanciados en el tiempo uno de otro. En ellos se pone de manifiesto de forma clara su capacidad de observación para los hechos naturales. Cronológicamente, el primero publicado (1854) es el correspondiente a las aves, en el que describe a las observadas habitualmente en la provincia de Sevilla, así como en algunas zonas de Huelva y Cádiz. Se trata, sin embargo, de un catálogo incompleto, ya que su autor reconoce que en la zona del Rocio y Almonte y en el coto de Doñana «existen multitud de zancudas y palmípedas que no he podido estudiar». En el catálogo Machado sigue el sistema de clasificación utilizado años antes por Villanova para las aves de la Albufera valenciana.

El libro correspondiente al catálogo de peces, referido a los de la costa de Cádiz y Huelva y al río Guadalquivir, vió la luz en 1857. Para su elaboración toma como base uno anterior, de 1817, elaborado por tres naturalistas: Antonio Cabrera, canónigo magistral de la catedral de Cádiz, Leonardo Flores, médico gaditano, y Félix Haenseler, alemán, farmacéutico y avecindado en Málaga. Machado cambió la clasificación de Linneo por la que había establecido Lis Bonaparte en su obra «Catálogo dei Pesci Europei», publicada en Nápoles en 1846. En la parte introductoria se lamenta de la explotación abusiva e incontrolada de los recursos marinos, en especial de la pesca de arrastre:

25. Revista Mensual de Filosofía, Literatura y Ciencias de Sevilla (1871), 3, 354. 
«...cuyas redes arrastradas por el buque abandonado a merced del viento recoge de los viveros naturales y de los fondos todos los peces de cualquier tamaño y edad que los habitan, sin considerar sea o no el tiempo de la propagación, y arrancando además y recogiendo las plantas marinas y mariscos que viven en aquellas profundidades y le sirven de alimento, de refugio o lugar de desove $\ldots \gg^{26}$.

La serie de catálogos aumentó en 1859 con el dedicado a los reptiles andaluces. Es quizás el más incompleto, como el mismo reconoce, por ser los reptiles «más difíciles de adquirirse que los otros vertebrados». En esta obra anuncia su intención de completar, en breve tiempo las catalogaciones, pero lo cierto es que hasta diez años mas tarde, en 1869 , no aparecería su catálogo de mamíferos de Andalucía.

Este último catálogo es bastante más ambicioso que los anteriores; en él se tratan diversas cuestiones de antropología, sociología y técnicas agropecuarias. Tras hacer en el prólogo un canto a las posibilidades agrícolas de Andalucía, pasa a considerar las distintas especies de mamíferos existentes en Andalucía, dedicando el mayor espacio al estudio del hombre andaluz. En el considera casi exclusivamente al campesino, tratando sus características antropométricas y sus costumbres. Cree que su alimentación es insuficiente, en especial la del jornalero, al basarse exclusivamente en los vegetales (gazpacho en verano y sopa de ajos en invierno), y considera que los jornales que se les pagaban eran de miseria. Opina sin embargo que la causa no es la distribución de la propiedad agrícola, sino el bajo rendimiento agrario que impide obtener un aceptable beneficio.

Durante los cuarenta años de magisterio en la Universidad sevillana recibió a diversos naturalistas extranjeros, a los que acompañó habitualmente en las excursiones que realizaron por los alrededores de Sevilla. Así, con el geólogo francés Delanoue, que llegó a Andalucía en 1868, exploró la comarca de Morón, en concreto la zona de la sierra de Esparteros y la llamada cañada de los Charcos, al pié de la cuesta del cortijo de Gatos, donde existían unos montecillos cónicos que a su modo de ver son volcanes de fangos, caracterizados por su lenta emisión de lodo, agua y vapores sulfhídricos. Machado los denomina con la voz italiana de «salssas», y los compara con los existentes en Sudamérica, donde reciben el nombre de volcancitos, aunque expone sus lógicas dudas acerca de la naturaleza de los mismos, dado que su comportamiento es muy diferente al de los volcanes clásicos que emiten materiales fundidos ${ }^{27}$.

26. MACHADO (1857), 2-5.

27. MACHADO (1869b), 12. 


\subsection{La difusión del darwinismo}

Machado fue uno de los más destacados pioneros de la difusión de las ideas evolucionistas de Charles Darwin en España. El primer artículo sobre este tema lo publicó en 1871, en la citada Revista de Ciencias, doce años después por tanto de la aparición de la primera edición de «El origen de las especies» (noviembre de 1859). No era ciertamente el primer escrito que se publicaba en España sobre el tema, ya que con anterioridad habían aparecido otros, pero insistiendo más en los aspectos sociológicos que en los científicos.

En este primer artículo, de gran claridad y sencillez, da inicialmente una idea general de la teoría de Darwin, que arrinconaba la tradicional teoría de la creación espontánea de las diferentes especies. Se refiere a continuación a los datos que sobre la evolución de los seres orgánicos suministra el estudio de los estratos de los terrenos sedimentarios: a lo largo de los siglos se va produciendo un aumento de la complejidad de dichos seres. No es posible la aparición simultánea de las diferentes especies porque las condiciones del planeta hace miles de siglos no eran aptas para la vida de aves y mamíferos, por el elevado contenido de anhídrico carbónico de la atmósfera.

Expone a continuaión Machado a sus lectores que la teoría de Darwin se basa en dos leyes fácilmente observables: la ley de selección natural, que conserva los seres de condiciones armónicas con los medios que los rodean y elimina a los que poseen desviaciones opuestas, y la ley de concurrencia vital o del combate perpetuo que todos los seres mantienen entre sí para alimentarse. Esta segunda ley es, según su opinión, una generalización de la ley de Malthus aplicada al mundo orgánico. Toda la exposición la acompaña de un gran número de casos prácticos, algunos tomados del entorno andaluz más cercano, posiblemente para hacer mas asequible las ideas y los conceptos al público selecto, pero no especializado, al que iba dirigida la revista.

$\mathrm{Al}$ año siguiente ampliaría Machado su información con un artículo titulado «Teoría de Darwin. Combate por la existencia», en el que refiere una serie de ejemplos sobre casos conocidos de lucha de especies por la supervivencia. En el mismo año publicó otro artículo, este titulado «Darwinismo», al principio del cual afirma:

«La teoría de Darwin, combatida por los reaccionarios de la ciencia, permanece sin embargo inalterable en sus bases fundamentales; las personas extrañas a la Geología niegan rotundamente las consecuencias de dichas teorías, a las cuales están apegados» ${ }^{28}$.

28. MACHADO (1872b), 523. 
Es posible que con estas palabras se refiera a la agria polémica desatada en Granada por la conferencia leída ese año en la apertura de curso en el Instituto de segunda enseñanza por el catedrático del mismo Rafael García Alvarez, en el que este habló y defendió las ideas evolucionistas, discurso que fue duramente combatido y condenado por el arzobispo de dicha ciudad ${ }^{29}$, lo que produjo un gran revuelo, circunscrito como es obvio a los ambientes intelectuales andaluces, ya que lo que hoy se denominaría gran público, sumido en el analfabetismo y en las estrechuras económicas, quedaba ajeno a aquéllas disquisiciones.

En otro artículo publicado ese mismo año aborda Machado el tema desde el punto de vista del hombre, en particular de la resistencia a las enfermedades, así como la evolución de los primates hasta la aparición del hombre, tema este que toca con suma prudencia:

«...cuando hablando de los cuadrumanos se atribuye el origen del hombre a las variaciones experimentales en los monos antropoideos, no se dice por esto que sean aquéllos seres nuestros progenitores, sino que se expresa solo una idea deducida de los principios de elección natural que Darwin demuestra en su teoría; en ella todos los seres aparecen sometidos a las leyes de una concurrencia severa, la cual hace desaparecer a los unos y conserva a los otros mientras las condiciones son a propósito para defenderse de las causas destructoras de la naturaleza» ${ }^{30}$.

En años sucesivos publicaría otros artículos sobre el tema evolucionista, y traduciría el libro de Herbert Spences sobre la misma cuestión. Con el tiempo, la polémica sobre el evolucionismo se iría apagando, aunque dicha teoría tardaría mucho en ser tolerada por los ambientes eclesiásticos y conservadores.

\subsection{Naturalistas posteriores a Machado}

En el momento de la marcha a Madrid de Machado Núñez la enseñanza de las Ciencias Naturales está plenamente establecida en Sevilla. La cátedra que dejó vacante la ocupó a fines de 1884 el profesor Salvador Calderón Arana, geólogo. Nacido en 1851 en Madrid, y con un importante curriculum realizado parcialmente en el extranjero, durante los años en que permaneció en Sevilla (marchó a Madrid en 1895) supo crear una escuela naturalista. Para ello se rodeó de un pequeño número de discípulos, con los que efectuaba excursiones por la provincia, en las cuales se llevaban a cabo pequeños trabajos

29. Boletín Eclesiástico del Arzobispado de Granada (1872), 28, 394.

30. MACHADO (1872b), 131. 
de prospecciones geológicas y de herborización. Entre los discípulos que, años después, cultivarían diversas ramas de la Historia Natural pueden citarse a Manuel Medina Ramos, Manuel Paul Arozarena, Romualdo González Fragoso (que llegaría a ser un afamado micólogo) y Francisco de la Barras de Aragón (que ocuparía la cátedra sevillana años después).

En sus diez años en Sevilla trabajó Calderón en diversos temas geológicos de carácter regional, en los que estudió los yacimientos minerales de la sierra de Peñaflor y los de diatomeas de Morón, así como los movimientos orogénicos del valle del Guadalquivir. Difundió además en Espan̄a las ideas geológicas de Suess y Neumayer, y fundó en Sevilla una sección local de la Sociedad Española de Historia Natural, la cual celebraba reuniones periódicas y en las que sus alumnos presentaban comunicaciones periódicas. Impartió también conferencias de divulgación en diversos centros culturales, entre ellos el Ateneo sevillano, y completó la colección de rocas y minerales del museo de la Universidad, estableciendo además un laboratorio de ensayos mineralógicos.

\section{CONCLUSIONES}

Aunque con carácter local, circunscrito a Sevilla y a su entorno regional, pero posiblemente extensible a otros lugares de España, la introducción de la ciencia moderna a lo largo del siglo XIX a la luz de los datos disponibles resumidos en el presente artículo, presenta las siguientes características:

1) La difusión ocurre con un apreciable retraso en relación con los países de la Europa occidental, especialmente en el caso de la Física, siendo menor este retraso en las otras materias consideradas.

2) Las ideas físicas tuvieron más oposición que las de las otras ciencias, a causa de su impacto sobre la tradicional doctrina escolástica de corte aristotélico y de las implicaciones teológicas de esta.

3) La Universidad fue incapaz de iniciar por sí misma las reformas necesarias para estudiar y difundir la ciencia moderna; los cambios que se llevaron a cabo fueron impuestos por las autoridades gubernativas, en especial con los planes de estudio de 1845 y 1857, y con el aumento de la dotación económica.

4) El cultivo de la ciencia, que se había dado exclusivamente durante el siglo XVIII en los centros extrauniversitarios, pasa a la Universidad a lo largo del siglo XIX. Dichos centros, de carácter cultural, experimentaron un marcado proceso de decadencia durante toda esta época. De forma paralela, la Iglesia pierde paulatinamente su influencia en la Universidad, y sus prerrogativas son absorbidas por el Estado. 
5) La modesta investigación científica que se realiza en este período corresponde de forma exclusiva a las Ciencias Naturales, en parte debido al bajo coste de este tipo de trabajo científico, basado principalmente en la observación.

6) La difusión del darwinismo científico se produjo entre 1871 y 1874, durante el llamado sexenio revolucionario.

7) Existió a lo largo del siglo XIX un contacto más intenso de lo que pudiera parecer con el extranjero. Muchos profesores de la Universidad sevillana (Machado, Campelo, Calderón, Manjarrés) estudiaron o trabajaron durante períodos variables de tiempo en el exterior, principalmente en Francia. También visitaron Andalucía numerosos naturalistas extranjeros, que fueron ayudados en sus trabajos por sus colegas españoles.

8) Fue muy frecuente que cada catedrático escribiera uno o más libros de texto para sus alumnos, quizás como un medio de incrementar sus menguados ingresos. También menudearon las traducciones de libros extranjeros.

9) No se consiguieron establecer estudios científicos completos de forma permanente. La Facultad de Ciencias, creada en 1857, sólo impartió hasta 1910 los cursos preparatorios (e incluso fue suprimida entre 1892 y 1895), mientras que la Escuela Industrial sólo funcionó durante unos doce años, y el Colegio de Farmacia tuvo una existencia aún más fugaz.

10) El mantenimiento de las enseñanzas científicas fue realizado de forma exclusiva por el Estado. No existieron ayudas de entidades o de particulares, sólo algunas donaciones de herbarios y de muestras de minerales.

11) El aumento de los presupuestos hacia la mitad del siglo propició la creación de los diversos gabinetes y el funcionamiento de los mismos.

\section{BIBLIOGRAFIA}

AGUILAR PIÑAL, F. (1961): «Fundación de la Sociedad Patriótica de Sevilla», Archivo Hispalense, $109,187-193$.

- (1969): La Universidad de Sevilla en el siglo XVIII, Publicaciones de la Universidad de Sevilla, Sevilla,

AJASSON DE GRANDSAQUE, J.B.F, y FOUCHE, J.M.L. (1840): Nuevo manual completo de Física y Meteorología (traducc. de F. Santos de Castro), Sevilla.

BARRAS DE ARAGON, F. (1944): Los naturalistas del distrito universitario de Sevilla, Anales Universidad Hispalense, Sevilla.

BOISSIER, E. (1839): Voyage botanique dans le midi de l'Espagne pendant I'annee 1837, Paris. 
BOUTELOU, C. (1827): Tratado de las flores, en que se explica el método de cultivar las plantas que sirven para adorno de los jardines, Madrid.

- (1842): Memoria de las plantas exóticas, Sevilla.

CANO PAVON, J.M. (1986): La Química en la Universidad de Sevilla. Estudio histórico, Archivo Hispalense, 212, 93.

- (1987): La ciencia experimental y la Universidad de Sevilla, Publicaciones Universidad de Sevilla, Sevilla.

COLMEIRO, M. (1847): Ampliación de la Botónica. Programa específicado ó resumen de sus lecciones, Sevilla.

- (1852): Investigaciones sobre la antigua madera conocida en Sevilla por el nombre de alerce, Comunicación a la Academia Sevillana de Buenas Letras, Sevilla.

- (1863): Tentativa sobre la Liquenología geográfica de Andalucía por D. Simón de Rojas Clemente, Madrid.

COLMEIRO, M. y BOUTELOU, E. (1854): Examen de las encinas y demás árboles de la Península que producen bellotas, con la descripción de los que se llaman mestos, Sevilla.

COZAR, P.J. y LISTA, A. (1841): Recuerdos del Doctor Mármol, Sevilla.

GAGO, R. (1982): La introducción e institucionalización de la Química en España: 1756-1808, Tesis doctoral en la Universidad de Granada.

GAGO, R. y CARRILLO, J.L. (1983): La introducción de la nomenclatura química y el rechazo de la teoría de la acidez de Lavoisier en España, Publicaciones Universidad de Málaga, Málaga.

GARCIA CUE, J.R. (1983): El hegelismo en la Universidad de Sevilla, Publicaciones Diputación Provincial, Sevilla.

- (1985): Aproximación al estudio del krausismo andaluz, Tecnos, Madrid.

GARNICA, A. (1988): Autobiografía de Blanco White (traducción comentada), Publicaciones Universidad de Sevilla, Sevilla.

Hermosilla molina, A. (1970): Cien años de Medicina sevillana, Publicaciones Diputación Provincial, Sevilla.

HERNANDEZ PACHECO, F. (1911): «Don Salvador Calderón y Arana y su labor científica», Boletín Sociedad Española de Historia Natural, II, 495.

HERRERA GARCIA, A. (1958): «Estudio histórico sobre el Real Colegio Seminario de San Telmo de Sevilla», Archivo Hispalense, 28, 253; 29, 47.

HERRERO, J. (1988): Los orígenes del pensamiento reaccionario español, Alianza, Madrid.

JACQUIER, F. (1784): Instituciones Philosophicae ad studia theologica potissimum accommodatur, Valencia.

LAZA PALACIOS, M. (1944): «Estudios sobre la flora andaluza», Anales Real Academia de Farmacia, 2, 157.

LIBES, A. (1827): El mundo físico y el mundo moral (traducción y prólogo de Manuel María del Mármol), Sevilla.

LOPEZ PIÑERO, J.M. (1969): La introducción de la cieneia moderna en España, Ariel, Barcelona.

LOPEZ PIÑERO, J.M.; GLICK, T.F.; NAVARRO BROTONS, V. y PORTELA MARCO, E. (1983): Diccionario histórico de la ciencia moderna en España, Península, Barcelona. 
MACHADO NUÑEZ, A. (1854): Catálogo de las aves observadas en algunas provincias de Andalucía, Sevilla.

- (1857): Catálogo de los peces que habitan en las costas de Cádiz y Huelva y en el Guadalquivir, Sevilla.

- (1859): Erpetología hispalensis, sen Catalogus Metodicus reptilium et amphibiorum in provintia hispallensi viventium, Sevilla.

- (1869a): Catálogo metódico y razonado de los mamíferos de Andalucía, clasificados según el método del Dr, Enrique Schinz, Sevilla.

- (1869b): «Excursión geológica a Morón y Conil», Revista mensual de Filosofía, Literatura y Ciencias de Sevilla, Sevilla, 1, 8.

- (1871): «Apuntes sobre la teoría de Darwin», Revista mensual..., 3, 461.

- (1872a): «Teoría de Darwin. Combate por la existencia», Revista mensual, 4, 3.

- (1872b): «Darwinismo», Revista mensual..., 4, 523.

- (1872c): «Apuntes para una memoria geognóstico-agrícola de la provincia de Sevilla», Revista mensual..., 4, 179 .

- (1872d): «Teoría de Darwin», Revista mensual..., 4, 129.

MANJARRES, R. (1860): Lecciones de Química Industrial Inorgáncia, Sevilla.

- (1862): Influencia de los fosfatos térreos en la vegetación y procedimientos más económicos para utilizarlos en la producción de cereales de la Península, Sevilla.

- (1896): El aceite de oliva. Su extracción, clasificación y refinación, Madrid.

MANJARRES, R. y LOSADA, V. (1863): Memoria acerca de la exposición internacional celebrada en Londres en 1862, Sevilla.

MARMOL, M.M. del (1817): Idea de los barcos de vapor o descripción de su máquina, relación de sus progresos e indicación de sus ventajas, Sanlúcar de Barrameda.

- (1825): Apuntes sobre los rumores ultimamente esparcidos acerca de la aproximación de la Luna a Ia Tierra, formados para desvanecer infundados temores, Sevilla.

- (1827): De Re Physica necessariores notiones quas indicabat, dialogisque digerebat, Sevilla.

- (1828a): El sistema de Copérnico puesto en verso, Sevilla.

- (1828b): Taquigrafía o arte de escribir deprisa como se habla, Sevilla.

- (1836): Ideas elementales de Física General, Sevilla.

- (1840): Apuntes o indicaciones de los más generales y precisos conocimientos de Química, hechos para auxiliar en los exámenes, Sevilla.

- (1843): Lógicae succus, Extractus dialogisque effusus, Sevilla.

MARTIN VILLA, A. (1886): Reseña histórica de Ia Universidad de Sevilla y descripción de su iglesia, Sevilla.

MENDEZ BEJARANO, M. (1922): Diccionario de escritores, maestros y oradores naturales de Sevilla y su actual provincia, Sevilla.

NUÑEZ RUIZ, D. (1975): La mentalidad positiva en España: desarrollo y crisis, Tucar, Madrid, 1975.

- (1977): El darwinismo en España, Castalia, Madrid, 1977.

PESET, M. y PESET, J.L. (1974): La Universidad española (siglos XVIII y XIX), Taurus, Madrid. 
PIQUER, A. (1785): Física modena, racional y experimental, Madrid.

SANTOS DE CASTRO, F. (1845): Nociones de Química, Sevilla.

- (1846): Curso elemental completo de Física experimental, Sevilla.

SELLES, M.; PESET, J.L. y LAFUENTE, A. (1988): Carlos II y la Ciencia de la Ilustración, Alianza, Madrid.

SPENCER, H. (1874): De la creación y la evolución (traducción de A. Machado Núñez), Revista mensual... 6, 385 .

VELAZQUEZ Y SANCHEZ, J. (1872): Anales de Sevilla. Reseña histórica de los sucesos políticos, hechos notables y particulares intereses de la tercera capital de la monarquía, metrópoli andaluza, Hijos de Fe, Sevilla. 